\title{
Effect of SERCA2a overexpression in the pericardium mediated by the AAV1 gene transfer on rapid atrial pacing in rabbits
}

\author{
B.N. Kuken ${ }^{1 *}$, A.N.W.E. Aikemu ${ }^{2 *}$, S.Y. Xiang ${ }^{3}$ and M.H.Y.T. Wulasihan ${ }^{4}$ \\ 1'Department of Cardiology, \\ The Second Affiliated Hospital of Xinjiang Medical University, Urumqi, Xinjiang, China \\ 2Department of Drug Analysis, Faculty of Pharmacy, Xinjiang Medical University, \\ Urumqi, Xinjiang, China \\ ${ }^{3}$ Intensive Care Unit, People's Hospital of Yanqi County, Yanqi County, Xinjiang, \\ China \\ ${ }^{4}$ Comprehensive Department of Cardiology, \\ The First Affiliated Hospital of Xinjiang Medical University, Urumqi, Xinjiang, China \\ *These authors contributed equally to this study. \\ Corresponding author: M.H.Y.T. Wulasihan \\ E-mail: 2920923487@qq.com
}

Genet. Mol. Res. 14 (4): 13625-13632 (2015)

Received May 9, 2015

Accepted August 19, 2015

Published October 28, 2015

DOI http://dx.doi.org/10.4238/2015.October.28.24

ABSTRACT. To study the effects of overexpression of the sarcoplasmic reticulum ATPase 2a (SERCA2a) gene on the activity and protein expression of SERCA2a after rapid atrial pacing (RAP) in New Zealand white rabbits. New Zealand white rabbits were randomly divided into a sham-operated group (group A), adeno-associated virus 1 (AAV1)/EGFP + atrial fibrillation (AF) model group (group B), or AVV1/SERCA2a + AF group (group C). The sham-operated group was used as a negative control. Each group consisted of 10 animals. Groups B and C were injected with $500 \mu \mathrm{L}$ of the AAV1-EGFP reporter gene and $500 \mu \mathrm{L}$ of the AAV1-SERCA2a target gene, respectively. Four weeks after AAV1-mediated gene transfer, the rabbits underwent $24 \mathrm{~h}$ of RAP to the right atrium. The animals were sacrificed and 
protein activity and protein expression in the myocardium were measured using the westernblot method. Four weeks after AAV1-mediated gene transfer, SERCA2a protein activity and expression were significantly higher in Group $C$ than in Groups $A$ and $B(P<0.05)$. RAP of the right atrium induced atrial fibrillation in rabbits, resulting in decreases in the activity and protein expression of SERCA2a. Pericardial AAV-1 mediated SERCA2a gene transfer resulted in the overexpression of SERCA2a, restoring SERCA2a activity and protein expression.

Key words: Atrial fibrillation; Sarcoplasmic reticulum $\mathrm{Ca}^{2+}$-ATPase; Rapid atrial pacing; Adeno-associated virus 1

\section{INTRODUCTION}

Atrial fibrillation (AF) is a common form of clinical arrhythmia. The incidence of AF is $1 \%$ among the general population, but increases with age and accounts for $10 \%$ of all arrhythmias in individuals $\geq 75$ years of age (Chen et al., 2012). As population age and other signs of social progress increase, so does the incidence of AF (Kannel et al., 1982). Atrial electrical remodeling is an important mechanism for the maintenance of $A F$, and abnormal intracellular calcium homeostasis is an important mechanism by which electrophysiological remodeling in the myocardium is initiated. Cytosolic $\mathrm{Ca}^{2+}$ is mainly released from the sarcoplasmic reticulum via ryanodine receptor 2 (RyR2). Abnormal expression of sarcoplasmic reticulum $\mathrm{Ca}^{2+}$ ATPase (SERCA) leads to an imbalance in the cytoplasm concentration of free $\mathrm{Ca}^{2+}$. This results in both systolic and diastolic dysfunctions. We used adeno-associated virus 1 (AAV1)/SERCA2a gene transfer to treat AF and to understand the mechanism behind any observed effects.

\section{MATERIAL AND METHODS}

\section{Materials}

Thirty adult New Zealand white rabbits, male and female, weighing 2.5-3 kg, were provided by the Experimental Animal Center of Xinjiang Medical University (China). The animals were of first grade quality (license number: SCXK (new) 2003-001). AAV1-EGFP and AAV1-SERC2Aa-EGFP were purchased from Beijing Vector Gene Technology Co., Ltd , Beijing, China. Titers were $1.0 \mathrm{x}$ $10^{12} \mathrm{vg}$ (viral genomes)/mL. SERCA2a mouse anti-rabbit polyclonal antibody was obtained from Abcam, Inc., USA. Bicinchoninic acid(BCA )Protein Assay Kits were obtained from Beijing Seitz Biotechnology Co., Ltd., Beijing, China.

\section{Grouping and modeling}

Experimental animals were randomly divided into three groups, with 10 animals in each group. The sham-operated group (Group A) received $500 \mu \mathrm{L}$ saline via transthoracic injection into the pericardial cavity. Pacing electrodes were implanted four weeks after injection, but no rapid atrial pacing (RAP) was performed. The AAV1/EGFP + AF model group (Group B) received $500 \mu \mathrm{L}$ AAV1-EGFP via transthoracic injection into the pericardial cavity. Four weeks later, the animals underwent RAP of the right atrium to induce AF. The AAV1/SERCA2a + AF group (Group 
C) received $500 \mu \mathrm{L}$ AAV1-SERCA2a-EGFP via transthoracic injection into the pericardial cavity. Four weeks later, RAP of the right atrium was used to induce AF.

\section{Ethics Statement}

The entire experiment was completed in the Experimental Animals Science Department in the Second Teaching Hospital of Xinjiang Medical University. Experimental protocols were reviewed and approved by the "Usage and Management Committee of Animals" of the First Teaching Hospital of Xinjiang Medical University, with the ethic lot number A-20100917001. The whole course of the experiment was finished under the supervision of this organization.

\section{Transthoracic injection of AAV1/SERCA2a into the pericardial cavity}

Animals were anesthetized by injecting $30 \mathrm{mg} / \mathrm{kg}$ body weight $3 \%$ sodium pentobarbital into the rabbit ear vein. Rabbits were placed on their left sides and the skin on the left side of the chest was disinfected using $75 \%$ ethanol. A $1-2 \mathrm{~cm}$ incision was made along the left side of the sternum midline to expose the sternum. The chest cavity was opened by an incision of approximately $5 \mathrm{~cm}$ at either the third or fourth intercostal space to expose the heart. The incision was gently held open using a retractor until the pericardium and beating heart were visible. Using ophthalmic tweezers, the pericardium was gently opened and a 32-gauge needle was inserted into the pericardial space. The needle was positioned where no blood could be drawn, and a slow bolus of AAV1 solution was injected, followed by closure of all incisions. Four weeks after gene transfer, rabbits were subjected to RAP. SERCA2a activity was then measured and immunohistochemistry was analyzed.

\section{RAP-induced AF model}

AF was induced by RAP according to the method described by Yang et al. (2012). Animals were anesthetized by injecting $30 \mathrm{mg} / \mathrm{kg}$ body weight $3 \%$ sodium pentobarbital into the rabbit ear vein. Rabbits were placed on their backs on a surgical table and intubated through the trachea for ventilator-assisted breathing. An incision was made at the center of the neck. The right internal jugular vein was separated and cut, and the superior end was ligated. Under ultrasound guidance, the pacing electrodes were inserted parallel to the ultrasound probe. A longitudinal slice was taken to verify that the position of the electrode was correct, and a latitudinal slice was taken to verify that the electrode was inside the right atrium. A LEAD2007 electrophysiology instrument was used (Sichuan Jinjiang Electronic, Chengdu, China) in continuous single stimulus mode to produce $24 \mathrm{~h}$ of RAP with a pacing rate of $600 \mathrm{times} / \mathrm{min}$, pulse width of $0.5 \mathrm{~ms}$, and intensity of $2 \mathrm{~V}$.

\section{Detection of AAV1/EGFP expression}

Four weeks after gene transfer, the animals in the AF-EGFP group were sacrificed. The heart, lungs, liver, and kidneys were removed, embedded in opti-mum cutting temperature compound (OCT) embedding medium, and sectioned into $5 \mu \mathrm{m}$ slices using a cryostat microtome. Slices were stretched on a glass slide, washed with phosphate buffer $(\mathrm{pH} 8.4)$, sealed with glycerol, and AAV1/EGFP expression was observed immediately using fluorescence microscopy. 


\section{Determination of rat myocardial SERCA2a activity}

Fifty milligrams of right atrial tissue was homogenized in $0.5 \mathrm{~mL}$ tissue homogenization buffer (Baoxin Biotech Co., Ltd., Beijing, China). The homogenate was centrifuged at $12000 \mathrm{rpm}$ for $5 \mathrm{~min}$, and the protein concentration of the supernatant was determined using the BCA method. Sample protein concentrations were adjusted to $1000 \mu \mathrm{g} / \mathrm{mL}$. The proteins were intubated in a $30^{\circ} \mathrm{C}$ water bath for $10 \mathrm{~min}$ and $20 \mu \mathrm{LATP}$ was added. Thirty seconds later, $20 \mu \mathrm{L}$ of the sample was added. After mixing for $45 \mathrm{~s}$, the OD values were measured. Once the curve was drawn, the maximal value was calculated.

\section{HE staining}

Tissue sections were dried in an oven for $20 \mathrm{~min}$, placed in xylene for $20 \mathrm{~min}$, followed sequentially by $100,95,80$, and $70 \%$ ethanol. Sections were then stained in hematoxylin for 5 min, rinsed with tap water, placed in a $1 \%$ solution of hydrochloric acid in ethanol for several seconds, and rinsed again with tap water for approximately $20 \mathrm{~min}$. Stained sections were placed into a $75 \%$ ethanol and eosin solution for a few seconds, then sequentially placed into 85,100 , and $100 \%$ ethanol for a few seconds for gradient dehydration. Finally, the sections were air-dried, neutral gum was placed onto the tissues, and the slices were covered with cover slides before examination under a light microscope.

\section{Immunohistochemical determination of SERCA2a expression in the right atrium}

Four weeks after gene transfer, the rabbits were sacrificed and right atrial myocardial tissue was collected. Tissues were fixed using $4 \%$ paraformaldehyde and were then embedded in paraffin and sliced into sections. The sections were incubated at room temperature for $2 \mathrm{~h}$ with a mouse anti-rabbit SERCA2a monoclonal antibody, then incubated with horseradish peroxidaselabeled secondary antibody for $1 \mathrm{~h}$. Color was allowed to develop for $5 \mathrm{~min}$. For each section, five fields were randomly selected at $400 \mathrm{X}$ magnification and cells that stained positive for the expression of SERCA2a were counted.

\section{Statistical analysis}

SPSS 16.0 was used for statistical analyses. Data are expressed as mean \pm SD. Comparisons among all groups were made using ANOVA. Comparisons between two groups were made using the Least significant difference (LSD) test. Differences with $P<0.05$ were considered to be statistically significant.

\section{RESULTS}

\section{Expression of AAV1/EGFP in the myocardium}

Green fluorescence in cardiomycetes was observed from the left and right atria after gene transfer four weeks. This indicates a high rate of AAV1 gene transfer.

\section{Expression of AAV1/EGFP in the lungs, liver, and kidney}

Lung, liver and kidney tissues showed a small amount of green fluorescence in group B. 
This method is a targeted method of administering AAV1 that results in minimal gene expression in other important tissues.

\section{SERCA2a activity assay}

The activity of SERCA2a in group B $\left(59.17 \pm 7.39 \mathrm{nmol} \cdot \mathrm{mg}^{-1} \cdot \mathrm{p}^{-1}\right)$ was significantly lower than those in group $A(105.33 \pm 10.56) \mathrm{nmol} /(\mathrm{mg} \cdot \mathrm{p})$ and group $C(96.17 \pm 5.19) \mathrm{nmol} /(\mathrm{mg} \cdot \mathrm{p})(P<$ 0.01). SERCA2a activity was not significantly different in groups $A$ and $C(P<0.01)$.

\section{HE staining}

As shown in Figure 1, group A animals had normal right atrial myocardial structure. The cells were arranged in neat rows, were wrapped in a small quantity of interstitial tissue, and had nuclei that were large and clear. In group B, cardiomycetes in the right atrial tissue were more disorganized than those in group $A$ and breaks in the muscle fiber could be observed. Connective tissue accumulated between the muscle fibers and there were gaps between myocardial cells. Group $\mathrm{C}$ had relatively normal myocardial cell structure, with neat organization, intact nuclei, and closely linked cardiomyocytes.
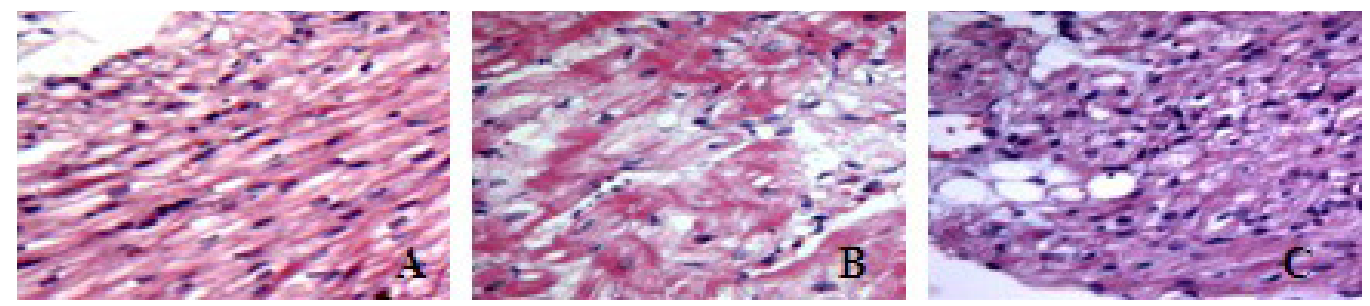

Figure 1. Myocardial organization as shown by hematoxylin and eosin staining in a sham-operated group (A), a group treated with AAV1/EGFP followed by atrial fibrillation (B), and a group treated with AAV1/SERCA2a followed by atrial fibrillation (C).

\section{Expression of SERCA2a in the right atrium}

Immunohistochemistry analysis indicated that, four weeks after gene transfer, SERCA2a was strongly expressed in the right atrial myocardium in group $C$. The expressions of SERCA2a in groups A, B, and C were $39.37 \pm 3.81 /$ field, $23.38 \pm 4.27 /$ field, and $58.75 \pm 4.71 /$ field, respectively. SERCA2a expression was greatest in group $A$, followed by group $C$ and group $B(P<0.01)$. Results are shown in Figure 2.
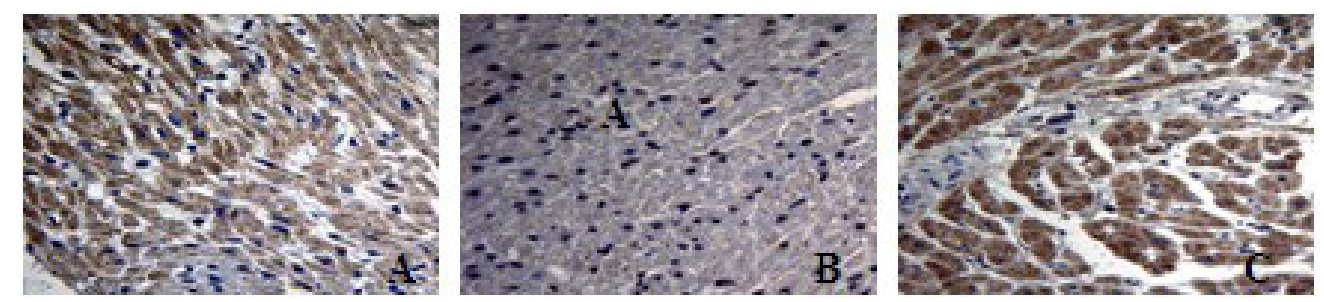

Figure 2. Expression of SERCA2a in the right atrial myocardium of a sham-operated animal (A), an animal treated with AAV1/EGFP followed by atrial fibrillation (B), and an animal treated with AAV1/SERCA2a followed by atrial fibrillation (C). 


\section{DISCUSSION}

Gene transfer is a potentially valuable treatment against cardiovascular disease. However, finding highly specific and safe gene transfer vectors is a major obstacle for its use as a clinical treatment. The method by which gene transfer is performed directly affects the efficiency of gene transfer. Current methods of gene transfer include cardiovascular injection, injection via peripheral vascular pathways, intramyocardial injection, and pericardial cavity injection. Cardiovascular injection (Meluzín et al., 2008), involves perfusion of the carrier solution via the aortic root/coronary pathway. This approach results in relatively high gene transfer efficiency, but can also produce target gene expression outside of cardiac tissues and may result in tissue damage. Injection via peripheral vascular pathways is easy and less invasive than cardiovascular injection, but carries low gene transfer efficiency and poor targeting that results in gene expression outside of the targeted cardiac tissues (Lei et al., 2009). Intramyocardial injection is a relatively easy method with targeted gene transfer and targeted gene expression in cardiac tissues (Gwon et al., 2001). However, the expression of the transferred genes is confined to a limited area around the injection site, and the injection itself can cause myocardial damage. Pericardial cavity injection is subdivided into transthoracic pericardial cavity injection and transabdominal pericardial injection. This method is operationally simple. Because the pericardial cavity is a closed space, the contact time between the gene vector and the heart is extended, resulting in improved gene expression in the heart. In this study, we used transthoracic pericardial cavity injection for in vivo gene transfer to cardiomyocytes. The overall procedure is less invasive than other available methods, conducive to rapid recovery, and permits repeat thoracotomy in case additional rounds of pericardial cavity gene transfer are needed. Wijffels (1995) put forward the idea of "Atrial electrical remodeling," which led to the "AF is the cause of AF" hypothesis. Atrial electrical remodeling mainly manifests as a reduction of the atrial effective refractory period (AERP), an increase in the AERP dispersion, and reduced conduction velocity, all of which contribute to the maintenance of AF. $\mathrm{Ca}^{2+}$ overload is an important mechanism behind the reduced AERP observed during AF, and significant changes in ion channel function are among the root causes of AF. As such, they may also be important targets in the treatment of AF. The calcium cycle in myocardiocytes proceeds as follows: inward current through L-type $\mathrm{Ca}^{2+}$ channels induce $\mathrm{Ca}^{2+}$ release by $\mathrm{RyR} 2$, which is found on the sarcoplasmic reticulum. The sarcoplasmic reticulum calcium uptake channel SERCA then recovers the cytoplasmic $\mathrm{Ca}^{2+}$ back into the sarcoplasmic reticulum. At the same time, the sodiumcalcium exchange channel on the plasma membrane transports a small portion of the $\mathrm{Ca}^{2+}$ out via the cell membrane to maintain calcium homeostasis in the cytoplasm. Changes in SERCA, including genetic mutations, abnormalities in protein expression, or dysfunctions in the protein can lead to arrhythmias. Our results show that pericardial injection can successfully transfer the SERCA2a gene into myocardiocytes and increase the expression of SERCA protein within the sarcoplasmic reticulum, thereby extending AERP and reducing the occurrence and development of AF. In humans, dogs and cats, 70 to $80 \%$ of the cytoplasmic $\mathrm{Ca}^{2+}$ is transported back to the sarcoplasmic reticulum by SERCA2a during the diastolic phase. Therefore, SERCA2a plays a key role in regulating the cytoplasmic $\mathrm{Ca}^{2+}$ concentration in myocardiocytes (Kawase et al., 2008). Our study used adenoviral gene transfer to overexpress SERCA2a in cardiomyocytes, resulting in an increase in SERCA2a activity and the upregulation of SERCA2a protein, which improved the ability of the sarcoplasmic reticulum to take up calcium and enhanced myocardial contractility. These results show that SERCA2a gene transfer has potential as a new therapeutic strategy against AF.

The recently published AFFIRM (2002) and RACE (Van Gelder et al., 2002) studies do not 
present any suitable treatments for AF. One of the main current treatments for AF, radiofrequency ablation, may cause serious complications and results in high recurrence rates over the long term. Researchers hope to improve atrial electrical remodeling through gene transfer in order to reverse the effects of AF without affecting other electrophysiological functions of the heart (Donahue et al., 2004; Gaborit et al., 2005). Treatment of AF through gene therapy requires changing the expression of the genes involved in AF in order to supplement or increase target protein expression for the improvement of cardiac electrophysiology and AF. There have been three primary approaches to international research of gene therapy for AF: conduction of the atrioventricular node to control AF (Kikuchi et al., 2005); regulation of myocardial cellular electrophysiology (Huang et al., 2007); and regulation of the function of atrial myocardial protein (Murata et al., 2004). The results of this study show that during AF, SERCA2a protein activity decreases and is downregulated. Therefore, SERCA2a overexpression via gene transfer may be a mechanism by which cardiac contractility can be improved. Our study did not address the long-term effects of SERCA2a on myocardial contractility, especially in the presence of the coexisting unfavorable factors that typically accompany AF. Further study of the effects of SERCA2a gene transfer on slow-pacing-induced chronic AF in large animals using the RAP-induced small animal AF model is required.

\section{Conflicts of interest}

The authors declare no conflict of interest.

\section{ACKNOWLEDGMENTS}

Research supported by grant \#81260036 from The National Natural Science Fund.

\section{REFERENCES}

Atrial Fibrillation Follow-up Investigation of Rhythm Management (AFFIRM) Investigators (2002). A comparison of rate control and rhythm control in patients with atrial fibrillation. N. Engl. J. Med. 347: 1825-1833.

Chen H, Zhang L, Yang SL, Sun J, et al. (2012). Influence of rapid atrial pacing on the electrophysiological changes in rats with atrial fibrillation. J. Chin. Pract. Diag. Ther. 26: 118-121.

Donahue JK (2004). Gene Therapy for Arrhythmias. Ann. NY Acad. Sci. 1015: 332-337.

Gaborit N, Steenman M, Lamirault G, Le Meur N, et al. (2005). Human atrial ion channel and transporter subunit geneexpression remodeling associated with valvular heart disease and atrial fibrillation. Circulation 112: 471-481.

Gwon HC, Jeong JO, Kim HJ, Park SW, et al. (2001). The feasibility and safety of fluoroscopy- guided percutaneous intramyocardial gene injection in porcine heart. Int. J. Cardiol. 79: 77-88.

Huang Y and Hou YM (2007). Research progress in gene therapy of atrial fibrillation . Chin. J. Cardiac. Pacing and Electrophys. 21: 108-110.

Kannel WB, Abbott RD, Savage DD, McNamara PM, et al. (1982). Epidemiologic features of chronic atrial fibrillation. N. Engl. J. Med. 306: 1018-1022.

Kawase $Y$ and Hajjar R J (2008). The cardiac sarcoplasmic/endoplasmic reticulum calcium ATPase: a potent target for cardiovascular diseases. Nat. Clin. Pract. Cardiovasc. Med 5: 554-565.

Kikuchi K, McDonald AD, Sasano T, Donahue JK, et al. (2005). Targeted modification of atrial electrophysiology by homogeneous transmural atrial gene transfer. Circulation 111: 264-270.

Lei J, Wu W, Xue SN, Zhen SX (2009). Effect of two transfection methods on foreign gene expression in myocardium. J. Sun. Yat. Sen. Univ. Med. Sci. 30: 56-58.

Murata M, Cingolani E, McDonald AD, Donahue JK, et al. (2004). Creation of a genetic calcium channel blocker by targeted gem gene transfer in the heart. Circ. Res. 95: 337-405.

Meluzín J, Vlaoin M, Groch L, Mayer J, et al. (2008). Intracoronary delivery of bone marrow cells to the acutely infarcted myocardium: optimization of the delivery technique. Cardiology 112: 98-106. 
Van Gelder IC, Hagens VE, Bosker HA, Kingma JH, et al. (2002). A comparison of rate control and rhythm control in patients with recurrent persistent atrial fibrillation. N. Engl. J. Med. 347: 1834-1840.

Yang SL, Zhou QN, Chen H, Xu XX, et al. (2012). Preventive role of atorvastatin in atrial fibrillation and its electrophysiologicai mechanism. J. Chin. Practical Diagn. Ther. 26: 449-451. 